\title{
L-Band Polarimetric Correlation Radiometer with Subharmonic Sampling
}

\author{
Rotbøll, Jesper; Søbjærg, Sten Schmidl; Skou, Niels
}

Published in:

Proceedings of the IEEE-2001 International Geoscience and Remote Sensing Symposium

Link to article, DOI:

10.1109/IGARSS.2001.976997

Publication date:

2001

Document Version

Publisher's PDF, also known as Version of record

Link back to DTU Orbit

Citation (APA):

Rotbøll, J., Søbjærg, S. S., \& Skou, N. (2001). L-Band Polarimetric Correlation Radiometer with Subharmonic Sampling. In Proceedings of the IEEE-2001 International Geoscience and Remote Sensing Symposium (Vol. 4, pp. 1571-1574) https://doi.org/10.1109/IGARSS.2001.976997

\section{General rights}

Copyright and moral rights for the publications made accessible in the public portal are retained by the authors and/or other copyright owners and it is a condition of accessing publications that users recognise and abide by the legal requirements associated with these rights.

- Users may download and print one copy of any publication from the public portal for the purpose of private study or research.

- You may not further distribute the material or use it for any profit-making activity or commercial gain

- You may freely distribute the URL identifying the publication in the public portal 


\title{
L-Band Polarimetric Correlation Radiometer with Subharmonic Sampling
}

\author{
Jesper Rotbøll, Member, IEEE, Sten Schmidl Søbjærg, Student Member, IEEE, \\ and Niels Skou, Senior Member, IEEE \\ Ørsted $\bullet$ DTU, Technical University of Denmark, \\ DK-2800 Lyngby, Denmark (e-mail: ns@oersted.dtu.dk).
}

\begin{abstract}
A novel L-band radiometer trading analog complexity for digital ditto has been designed and built. It is a fully polarimetric radiometer of the correlation type and it is based on the sub-harmonit: sampling principle in which the L-band signal is directly sampled by a fast $A$ to $D$ converter at a frequency well below L-band. Stability has been a design driver, and the instrument is intended for airborne measurements of polarimetric sea signatures.
\end{abstract}

\section{INTRODUCTION}

An L-band $(1.4(\mathrm{GHz})$ radiometer system, based on novel interferometric tectniques, and known under the acronym SMOS (soil moisture ocean salinity), has been selected as one of ESA's Earth Explorer Opportunity Missions. Measuring the L-band brightness temperature over the oceans enables a determination of sea salinity.

The brightness temperature sensitivity to salinity is typically (open ocean with high salinity) $\Delta \mathrm{T}_{B} / \Delta \mathrm{S}=1 \mathrm{~K} / \mathrm{psu}$ (vertical polarization, $50^{\circ}$ incidence angle). Hence, to find salinity to the $0.1 \mathrm{psu}$ level requires radiometric measurements to better than $0.1 \mathrm{~K}$ and knowledge concerning the influence of other effects to the same level. Sea surface temperature is one of these effects, and it must be measured and corrected for. This is doable, however, as it is a well modeled and understood effect, and the ocean temperature is well monitored. But, the knowledge about the influence of wind on the brightness temperature has been identified as insufficient. Measurements and model work have concentrated on higher frequencies, and very little knowledge about any polarimetric signature - i.e. the brightness temperature dependence on azimuth viewing angle relative to the wind direction - exist. The present paper describes actions taken with the aim of helping to fill this void by designing, and developing a suitable L-band polarimetric radiometer and antenna system, and carry out airborne campaigns in order to experimentally determine the radiometric response of the ocean to wind speed and direction relative to the observing direction.

\section{POLARIMETRY AND STOKES PARAMETERS}

The (brightness) Stokes vector is:

$$
\overline{T_{B}}=\left(\begin{array}{c}
I \\
Q \\
U \\
V
\end{array}\right)=\left(\begin{array}{c}
T_{V}+T_{H} \\
T_{V}-T_{H} \\
T_{45^{\circ}}-T_{-45^{\circ}} \\
T_{1}+T_{r}
\end{array}\right)=\frac{\lambda^{2}}{k \cdot z}\left(\begin{array}{c}
\left\langle E_{V}^{2}\right\rangle+\left\langle E_{H}^{2}\right\rangle \\
\left\langle E_{V}^{2}\right\rangle-\left\langle E_{H}^{2}\right\rangle \\
2 \operatorname{Re}\left\langle E_{V} E_{H}^{*}\right\rangle \\
2 \operatorname{Im}\left\langle E_{V} E_{H}^{*}\right\rangle
\end{array}\right)
$$

where $\mathrm{z}$ is the impedance of the medium in which the wave propagates, $\lambda$ is the wavelength and $k$ is Boltzmanns constant. $T_{V}$ is the vertical and $T_{H}$ the horizontal brightness temperature. $\mathrm{T}_{45^{\circ}}$ and $\mathrm{T}_{-45^{\circ}}$ represent orthogonal linearly polarized measurements skewed $45^{\circ}$ with respect to normal and $T_{1}$ and $T_{r}$ refer to left-hand and right-hand circular polarized quantities. I represents the total power, and $\mathrm{Q}$ the difference of the vertical and horizontal power components. The first and second Stokes parameters are measured using vertically and horizontally polarized radiometer channels, followed by addition or subtraction of the measured brightness temperatures.

The third Stokes parameter can be found as the real part of the cross correlation of the vertical and horizontal electrical fields. The fourth Stokes parameter can be measured as the imaginary part of the cross correlation of the vertical and horizontal electrical fields.

\section{THE POLARIMETRIC RADIOMETER}

The L-band polarimetric radiometer measures all four parameters of the Stokes vector simultaneously, using the correlation principle. Further information on different radiometer configurations for measuring polarimetric signatures can be found in [1]. The fundamental difference between a traditional polarimetric correlation radiometer (Figure 1) and a subharmonic sampling polarimetric correlation radiometer (Figure 2) is the use of fast A/D converters, not only for detection and correlation (in order to derive the Stokes vector), but also for frequency shift from RF to baseband (subharmonic sampling).

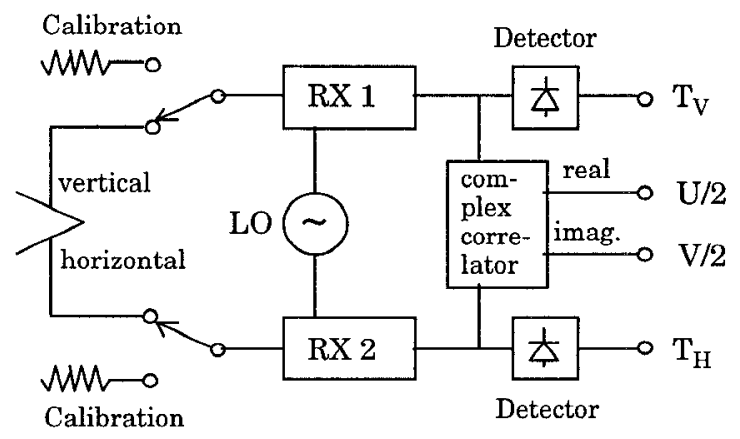

Figure 1: Basic correlation radiometer. 


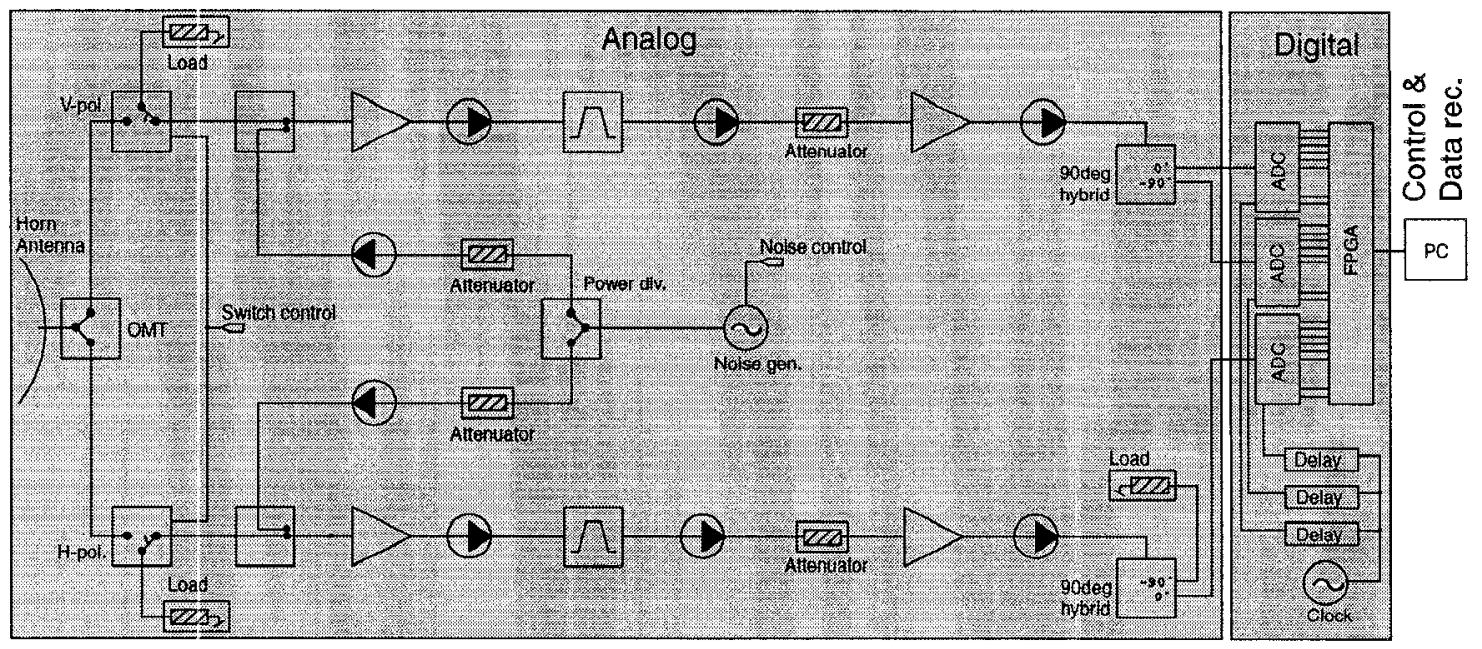

Figure 2: Subharmonic sampling correlation radiometer.

\section{A. The Analog Part}

The antenna must be large measured in wavelengths in order to ensure a reasonably narrow angular beamwidth, hence a small range of incidence angles over which the measurements are integrated. Due to the long L-band wavelength this results in a physically large antenna. For the present purpose circle flights with the antenna looking out to the side of the aircraft is the optimum solution. This way different circles with different bank angles will directly reveal the azimuthal signatures as functions of incidence angles, provided the antenna is mounted properly. The side-looking geometry limits the aperture to $90 \mathrm{~cm}$ taking advantage of the aft parachute doors of a C-130 airplane. $90 \mathrm{~cm}$ aperture corresponds to a $15^{\circ}$ beamwidth, which is considered acceptable. A large horn with a $90 \times 90 \mathrm{~cm}$ square aperture and a length of $200 \mathrm{~cm}$ has been constructed. This antenna option has been selected due to simplicity and good performance - especially concerning loss, which is of prime importance in the present case. An ortho-mode transducer enables retrieval of $\mathrm{H}$ and $\mathrm{V}$ polarizations.

Figure 2 shows the analog radiometer section, consisting of two total-power radiometer channels, and two calibration circuits for gain and correlator calibration. Each channel has a total noise figure $\mathrm{NF}=1.4 \mathrm{~dB}$, corresponding to a noise temperature $\mathrm{T}_{\mathrm{N}}=119 \mathrm{~K}$ (system temperature stabilized at 313 $\mathrm{K})$, and a filter bancwidth $\mathrm{BW}=27 \mathrm{MHz}(1400 \mathrm{MHz}-1427$ $\mathrm{MHz}$ ). Thus the sensitivity is $\Delta \mathrm{T}=0.1 \mathrm{~K}$ for $1 \mathrm{sec}$. integration period, when viewing a $300 \mathrm{~K}$ target.

As the $\mathrm{A} / \mathrm{D}$ conversion is not only a media conversion but also introduces a frequency shift as mentioned above, special requirements to the analog RF-filter arise, due to the risk of aliasing from other frequency bands. Figure 3 shows the spectral behavior of a subharmonic sampling, using a sampling frequency $\mathrm{f}_{\mathrm{s}}=63.5 \mathrm{MHz}$, a filter bandwidth $\mathrm{BW}=$
$27 \mathrm{MHz}$ and an overall system bandwidth $\mathrm{BW}_{\text {sys }} \approx 300 \mathrm{MHz}$, limited by the OMT. The chosen $f_{s}$ will be discussed in the next section. We here notice that four harmonics of $f_{s}$ fall within the system bandwidth $\mathrm{BW}_{\text {sys }}$, causing heavily aliasing at IF level in a case with poor RF-filter stopband attenuation, because every harmonic of $f_{s}$ converts noise around it to baseband (IF). The filter stopband attenuation is set to $60 \mathrm{~dB}$ at the frequencies $1390 \mathrm{MHz}$ and $1437 \mathrm{MHz}$, due to the risk that interference signals outside the limited filter bandwidth add up at baseband (IF), but more importantly because $60 \mathrm{~dB}$ stopband attenuation is standard in a traditional superheterodyne correlation radiometer, using two filters (RF and IF).

Another aspect to consider when designing a subharmonic sampling radiometer is the analog RF gain. In a traditional superheterodyne radiometer the gain can be divided between the RF- and the IF-circuit. This is not the case in a subharmonic sampling radiometer where the overall gain is at RF-level, with a potential risk of generating feed-back problems, e.g. from the analog output, through the air and into the antenna. With an overall gain of approximately $100 \mathrm{~dB}$ even a small leak can be fatal. Standard SMA-connectors are used to connect the RF-components in the radiometer channels, right from the antenna output to the $A / D$ converter printed circuit board (PCB). The leak in a standard SMAconnector is specified to be below $-100 \mathrm{~dB}$, and the antenna back lobe level is approximately $-25 \mathrm{~dB}$ (the antenna is pointing away from the radiometer). This provides at least $135 \mathrm{~dB}$ isolation, which is sufficient to avoid feed-back. Experience has, however, shown that insufficient shielding of the digital A/D converter PCB can cause problems, as even a short line on the PCB will act as a radiating antenna at Lband. 

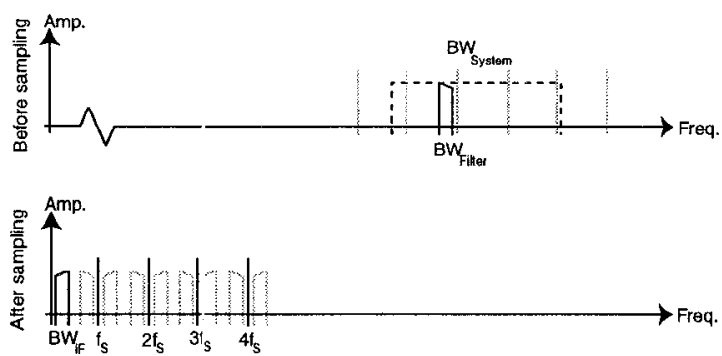

Figure 3: Spectral behavior of a subharmonic sampling.

Important is not only the isolation between the output and input of the analog channels, but also the isolation between the two channels. The result of poor isolation between the channels is correlation, i.e. offsets on the output of the correlator (third and fourth Stokes parameters), as the leak signal and the original signal will correlate. Two circuits, the correlated noise injection calibration circuit and the antenna (actually the OMT), provides a direct path between the two radiometer channels, and are therefore especially critical. In the correlated noise injection calibration circuit an isolation of approximately $72 \mathrm{~d} 3$ is obtained. The OMT has an isolation of approximately $40 \mathrm{~dB}$, which could easily be improved by adding an isolator in each channel, however resulting in higher loss and degraded sensitivity.

As in any other correlation radiometer phase coherence between the two receivers is required, in order to measure the complex correlation between the input signals. In the analog part of the system the components are matched in a way that the overall phase difference between the two channels is less than $5^{\circ}$. A phase difference will cause a shift of $U$ signal into the $\mathrm{V}$ channel and $\mathrm{V}$ into $\mathrm{U}$. Fine tuning can be done in the digital part of the radiometer (described in the next section III- $B$ ).

The analog part of the radiometer includes two calibration circuits, a noise diode arrangement for correlator calibration, and coax switches with heated loads for total power channel calibration. The calibration procedure is described in section III- $C$. The last component in the analog part of the radiometer is a quadrature hybrid, used for generating in-phase and $90^{\circ}$ out-of-phase signals for the correlator.

\section{B. The Digital Part}

Using modern arralog-to-digital converters (ADC), it is possible to sample the L-band signal directly, without downconversion to base-band. The Maxim MAX104 ADC is an example of the available technology, and it provides an analog $3 \mathrm{~dB}$ bandwidth better than $2 \mathrm{GHz}$ and sampling speeds better than $1 \mathrm{GHz}$, providing at least 6.5 effective bits over all the frequency range.

From the general sampling theory, it is well known, that the periodic sampling of an analog signal returns a spectrum, which is described thy the convolution of the original signal's spectrum and a train of infinitesimally short pulses, spaced by the sampling frequency, $f_{s}$. This means, that the resulting base-band spectrum will be formed as an infinite sum of the original base-band spectrum, added to the specters around $\mathrm{n}$ times the sampling frequency, where $\mathrm{n}$ is an integer. Traditionally this leads to a low pass filter applied in front of the sampling circuit to limit the signal bandwidth, but using an ADC with high analog bandwidth, a narrow band RF/IF signal may be digitized directly, according to Figure 3 . This process is known as subharmonic sampling, and the figure shows, that the sampled spectrum contains the same information as the original signal. The choice of sampling frequency, $f_{s}$, however, is essential for the preservation of information. The general sampling theorem, stating that $\mathrm{f}_{\mathrm{s}} \geq 2$. BW, must be fulfilled, but additionally it must be observed, that $n \cdot f_{s}$, where $n$ is an integer, does not falls within the pass-band of the original signal. This will result in overlap from the two side-bands, causing loss of information. For the present radiometer, covering the bandwidth from $1400 \mathrm{MHz}$ to $1427 \mathrm{MHz}$, a sampling frequency of $63.5 \mathrm{MHz}$ has been chosen, placing the sampled spectrum from $3 \mathrm{MHz}$ to $30 \mathrm{MHz}$.

The subharmonic sampled signal may be regarded as equivalent to a down converted, sampled signal, providing the same properties of linearity and standard deviation. The linearity depends on the level of saturation, i.e. the $\mathrm{min} / \mathrm{max}$ values for the ADC output. For the MAX104, the input range is $\pm 250 \mathrm{mV}$, approximately equal to $1 \mathrm{dBm} @ 50 \Omega$. Choosing the input signal level to $-10 \mathrm{dBm}$, the effect of unlinearity will be negligible.

For the standard deviation, it can be shown [2], that the standard deviation of a radiometer signal, sampled with an infinite number of bits, will be equal to the standard deviation of the unsampled signal, if the whole bandwidth is preserved during the sampling. The effect of quantization may be regarded as the addition of a small noise signal with the power $\mathrm{e}_{0}{ }^{2} / 12$, where $\mathrm{e}_{0}=500 \mathrm{mv} / 2^{8}$ for the 8 bit MAX104/108. At $50 \Omega$ this is equal to $-52 \mathrm{dBm}$, which is negligible.

The complete digital radiometer basically consists of three parallel correlators, where the first and second Stokes parameters are formed by selfcorrelation of the horizontal and vertical brightness temperatures. The third correlator calculates the complex correlation between the two polarizations, giving the third and fourth Stokes parameters.

An important issue in the implementation of correlators is that a DC component in the input signal will severely influence the output. The A/D converters themselves provide a DC offset adjustment pin, and using input signals without DC, the mean voltage value can be calculated from the radiometer data, and a simple regulation loop may be applied, feeding the adjustment pin from a small digital-to-analog converter. Alternatively the DC component can be removed in the digital circuit, using a DC block filter, when the sampled signal does not cover all the bandwidth down to DC. As we here use frequencies from $3 \mathrm{MHz}$ to $30 \mathrm{MHz}$, the digital DC removal has been implemented in the present system. 
A final aspect to consider in the implementation of the subharmonic sampling is the exact alignment of sampling times for the three ADC's, relating to the phase of the input RF signal. The delays are adjusted using dedicated delay circuits, digitally programmable in a $\pm 2 \mathrm{~ns}$ range and with a possibility of sub-psec. precision using an analog input. The delay setting is calculated using the correlators and the noise diode of the radiometer. When applying in-phase correlated noise to the two radiometer channels, the real output from the digital correlator will calculate the level of correlation, while the imaginary part should return a zero result, when the $\mathrm{ADC}$ 's are perfectly aligned. Likewise, the correlation between the 0 degrees and the 90 degrees shifted inputs from the same channel should be zero. Implementing a special test mode to calculate the two zero correlations, a simple regulation of the sampling delays can be done before final assembly of the system.

\section{Overall system considerations}

Aspects such as stability and absolute accuracy are important when you design a microwave radiometer. In the present L-band radiometer system this is obtained by in-flight calibration and temperature stabilized design.

The system is temperature stabilized using a regulator and heaters to heat a box to $313 \mathrm{~K}$ within a few tenths of a degree. The analog part of the radiometer is then located inside the box, without having direct thermal contact to the temperature regulated box, i.e. a box in a box principle. The digital PCB is temperature stabilized in a simple way, using the heat generated by the A/D converters, for heating the shielded box in which the PCB is located. Data from several internal (analog and digital box) and external (OMT and RF-cables) temperature sensors is stored on the PC, making temperature compensation of the data possible.

Using the coax switches with the heated loads before and after each track ensures good absolute accuracy of the detection (total power) channels. The coax switches and the heated loads can also be used for injection of uncorrelated noise in the two receiver channels, making offset calibration of the correlator possible. Furthermore a noise generator can inject correlated noise into the two receiver channels, and the degree of correlation at the receiver output can be precisely calculated using the measurements from the detection channels. Hence absolute calibration of the radiometer is possible, even during flight.

\section{THE EXPERIMENTS}

Two flights have been carried out over Danish waters during 2001. During both flights, conditions were favorable for microwave radiometer measurements, with no heavy clouds or rain. The data recorded has not yet been processed, i.e. calibrated and corrected for airplane attitude (e.g. roll and pitch), yet it is clear, that there was no interference. Preliminary review indicates a small polarimetric (U and V) response, if any.

\section{FUTURE PLANS}

The L-band polarimetric correlation radiometer is designed with field programmable gate arrays (FPGA) in the digital front-end, giving full flexibility for reconfiguration.

- An increase in the RF bandwidth would result in a better sensitivity, but also a higher probability of interference signals. In the digital domain, a second filter can provide additional modification to the frequency band selected by the RF-filter, allowing selective suppression of possible interference signals.

- A special time-domain filtering may be applied to avoid pulsed interference signals. Making the integration periods shorter, and evaluating the power level of each integration period, will enable rejection, if the signal level changes significantly beyond the level of radiometric noise. The short integration period can then be followed by a longer integration to e.g. $8 \mathrm{msec}$. (to reduce the data amount), keeping the number of rejected short integration periods in mind.

- A phase shift like the one provided by the two analog quadrature hybrids, can also be made digitally, or alternatively a full digital $\mathrm{I} / \mathrm{Q}$ demodulaton can be implemented. A digital solution would reduce the number of components, analog as well as digital, i.e. reduce size, weight, power consumption and cost.

\section{CONCLUSIONS}

A new polarimetric correlation radiometer has been designed, build and operated. As the radiometer employs subharmonic sampling, special considerations regarding the analog and digital circuit has been discussed. The system features several advantages as compared with a traditional superheterodyne: weight, size and flexibility for reconfiguration. The system is being used for measuring the brightness temperature over the ocean in experiments carried out in 2001, in order to determine the influence of the wind at the L-band brightness temperature. Preliminary results seems very encouraging and will soon be the subject of proper publications.

\section{REFERENCES}

[1] Niels Skou, Brian Laursen and Sten Søbjærg: "Polarimetric Radiometer configurations: Potential Accuracy and Sensitivity", IEEE Trans. Geo. Remote Sensing, vol 37, pp. 2165-2171, sep.99

[2] Thompson, Moran, Swenson: 'Interferometry and Synthesis in Radio Astronomy"; John Wiley \& Sons, 1986 